\title{
Comparison of small-volume tubes and vacuum blood tubes for complete blood count
}

\author{
Kubranur Unal
}

Department of Biochemistry, Polatlı Public Hospital, Ankara, Turkey

\begin{abstract}
Objectives: A complete blood count $(C B C)$ is one of the most commonly requested clinical laboratory tests. Vacuum blood tubes are used routinely, and now, new small-volume tubes (SVTs) containing dipotassium ethylenediaminetetraacetic acid (K2EDTA) are also in use. The aim of this research was to compare SVTs with vacuum blood tubes for use in a CBC.

Methods: Venous blood samples were taken from 40 healthy volunteers and were collected in BD Vacutainer (Becton, Dickinson and Company, Franklin Lakes, NJ, USA) K2EDTA tubes and BD Microtainer (Becton, Dickinson and Company, Franklin Lakes, NJ, USA) K2EDTA tubes. CBC parameters were analyzed using an ABX Pentra DF 120 device (Horiba, Ltd., Kyoto, Japan).

Results: Red blood cells (RBC), hemoglobin (HGB), hematocrit (HCT), mean corpuscular hemoglobin (MCH), mean corpuscular hemoglobin concentration (MCHC), and basophil (BASO) levels were found to be statistically significantly higher, while platelet (PLT) levels were determined to be statistically significantly lower in the SVT analyses compared with those of the vacuum blood tubes. When the percentage difference was compared with the total allowable error, $\mathrm{RBC}, \mathrm{HGB}, \mathrm{HCT}, \mathrm{MCH}, \mathrm{MCHC}$, red cell distribution width, white blood cell count, neutrophil, lymphocyte, monocyte, eosinophil, and BASO values demonstrated a general trend of positive bias, while PLT values demonstrated a general trend of negative bias on a Bland-Altman bias plot.

Conclusion: The results of this study demonstrated that venous blood samples from SVTs generated a significant bias in the $\mathrm{CBC}$ results. In addition, clotted samples were observed more frequently among those collected in SVTs in this study. The design of SVTs may have an important and detrimental effect on the accuracy and efficacy of the results of a CBC.

Keywords: Blood collection tube, complete blood count, dipotassium ethylenediaminetetraacetic acid, performance comparison, small-volume tubes
\end{abstract}

$\mathrm{T}$ he complete blood count $(\mathrm{CBC})$ is one of the most commonly requested clinical laboratory tests [1]. Accurate measurement of the $\mathrm{CBC}$ is essential for the correct diagnosis, treatment, and follow-up of patients with hematological disorders. These measurements are now almost fully automated, and the right interpretation of the clinical significance of the results requires knowledge of the pre-analytical phases [2].

The blood collection tubes used for a CBC should contain anticoagulant ethylenediaminetetraacetic acid (EDTA), according to the recommendation of the Clinical \& Laboratory Standards Institute (CLSI) [3]. EDTA salts are used for the anticoagulation of whole blood for hematological analyses because the cellular components of the blood are well preserved by EDTA [4]. Today, a CBC is typically performed with whole blood collected in tubes containing dipotassium EDTA (K2EDTA) as an anticoagulant. Blood collection tube additives and components are an important component of the pre-analytical phase in terms of a potential influence on laboratory test results. Although

Address for correspondence: Kubranur Unal, MD. Department of Biochemistry, Polatlı Public Hospital, Ankara, Turkey Phone: +90 31263030 30-11 02 E-mail: dr.kubranur_unal@outlook.com ORCID: 0000-0001-7940-4590

Submitted Date: December 07, 2018 Accepted Date: January 02, 2019 Available Online Date: January 15, 2019

${ }^{\circ}$ Copyright 2018 by International Journal of Medical Biochemistry - Available online at www.internationalbiochemistry.com OPEN ACCESS This work is licensed under a Creative Commons Attribution-NonCommercial 4.0 International License. 
the influence of blood collection tubes on laboratory tests is usually ignored, the correct choice of blood collection tube is essential [5]. Another issue is that the amount of additive placed in a tube is designed for a certain volume of blood. If less blood than required is taken, the excess amount of additive has the potential to adversely affect the accuracy of the test results [6].

Vacuum blood tubes are frequently used in routine practice, and now small-volume tubes (SVTs) containing K2EDTA have been also manufactured. SVTs are suitable for taking samples and the transport and processing of samples in cases where only a small amount of blood is required [7]. To the best of our knowledge, there is no study in the literature that evaluates BD Microtainer K2EDTA and BD Vacutainer K2EDTA tubes (Becton, Dickinson and Company, Franklin Lakes, NJ, USA) using the CBC. The aim of this study was to compare SVTs with vacuum blood tubes for use in performing a $C B C$.

\section{Materials and Methods}

\section{Subjects and study design}

A total of 40 healthy adult volunteers ranging in age between 20 and 55 years were included in this study. All of the participants provided written, informed consent. This study was approved by the Ethics Committee of Ankara Polatlı State Hospital.

SVTs are designed primarily for capillary blood collection [8]. However, in this study, venous blood collection was used for both tubes. Two venous blood samples from each volunteer were taken from a single cubital region and were collected into BD Vacutainer K2EDTA tubes and BD Microtainer K2EDTA tubes:

1. BD Vacutainer K2EDTA tube: $3 \mathrm{~mL}$ (volume), $13 \times 75 \mathrm{~mm}$ (size), 5.4 mg EDTA (additive) (Catalog number: 368856).

2. BD Microtainer K2EDTA tube: $250-500 \mu \mathrm{L}$ (volume), $10 \times 35$ $\mathrm{mm}$ (size), $0.8 \mathrm{mg}$ EDTA (additive) (Catalog number: 365975).

The tubes were filled to capacity. After the blood samples were taken, all of the tubes were immediately mixed with gentle $180^{\circ}$ inversion to avoid microclotting. The samples were analyzed 15 to 30 minutes after venipuncture. All of the measurements were performed in duplicate.

\section{Methods}

The cellular count result of the ABX Pentra DF 120 hematological analyzer (Horiba, Ltd., Kyoto, Japan) using electronic impedance variation in 2 different K2 EDTA tubes was evaluated at the Ankara Polatlı Public Hospital Department of Biochemistry Central Laboratory. The following analytes were measured in both tubes for each of the 40 subjects: complete blood count (CBC), red blood cell (RBC), hemoglobin (HGB), hematocrit $(\mathrm{HCT})$, mean corpuscular volume (MCV), mean corpuscular hemoglobin $(\mathrm{MCH})$, mean corpuscular hemoglobin concentration (MCHC), red cell distribution width (RDW), platelet (PLT), white blood cell (WBC) count and differential, including neutrophils (NEU), lymphocytes (LYMPHO), monocytes (MONO), eosinophils (EOS), and basophils (BASO). Internal and external quality control data were checked before the study.

Due to the insufficient volume of the SVTs, the tubes were not entered into a stability test.

\section{Statistical analysis}

The data were processed using PASW Statistics for Windows, Version 18.0 (SPSS Inc., Chicago, IL, USA) and GraphPad Prism version 7 (GraphPad Software, San Diego, CA, USA). The Kolmogorov-Smirnov test was performed to assess normality. The descriptive statistics of continuous variables were expressed as mean \pm SD or median (min-max). A paired samples t-test was also performed to compare the results for parametric variables and a Wilcoxon's signed-rank test was applied to nonparametric continuous variables. A $p$ value of $<0.05$ was considered statistically significant.

The difference in concentration between tubes at a single time point was calculated as mean difference $(\%)=$ (concentration BD Microtainer K2EDTA tubes-concentration BD Vacutainer K2EDTA tubes)/concentration BD Vacutainer * 100. The mean difference (\%) was compared with the total allowable error (TAE), which was based on the within-and between-subject coefficients of variation provided by Ricos et al. [9] for each of the analytes measured.

Correlation and linear regression analysis were used to assess the difference between results obtained in 2 different types of blood collection tubes. The guide proposed by Evans [10] to determine the absolute value of $r$ was used to evaluate the strength of the correlation in this study. A 95\% confidence interval $(\mathrm{Cl})$ for the slope and intercept was calculated from the standard error of regression. Bland-Altman plots were generated to ascertain areas of bias between blood collection tubes [11]. The mean difference was also assessed with Bland-Altman plots and then compared with the TAE for bias.

This study was conducted using the CLSI guideline for method comparison and bias estimation using patient samples as a reference [12].

\section{Results}

The comparison of the results obtained from SVTs with those of vacuum blood tubes is shown in Table 1. When the mean and median values of the results of BD Microtainer K2EDTA tubes were compared with BD Vacutainer K2EDTA tubes, the RBC, $\mathrm{HGB}, \mathrm{HCT}, \mathrm{MCH}, \mathrm{MCHC}$, and BASO levels were found to be statistically significantly higher, while the PLT levels were found to be statistically significant lower. There was no statistically significant difference between the 2 groups in terms of MCV, RDW, WBC, NEU, LYMPHO, MONO, and EOS level. The observed differences were clinically significant since the mean difference at baseline for RBC, HGB, HCT, MCH, MCHC, PLT, and BASO exceeded the current desirable quality specifications [9]. 


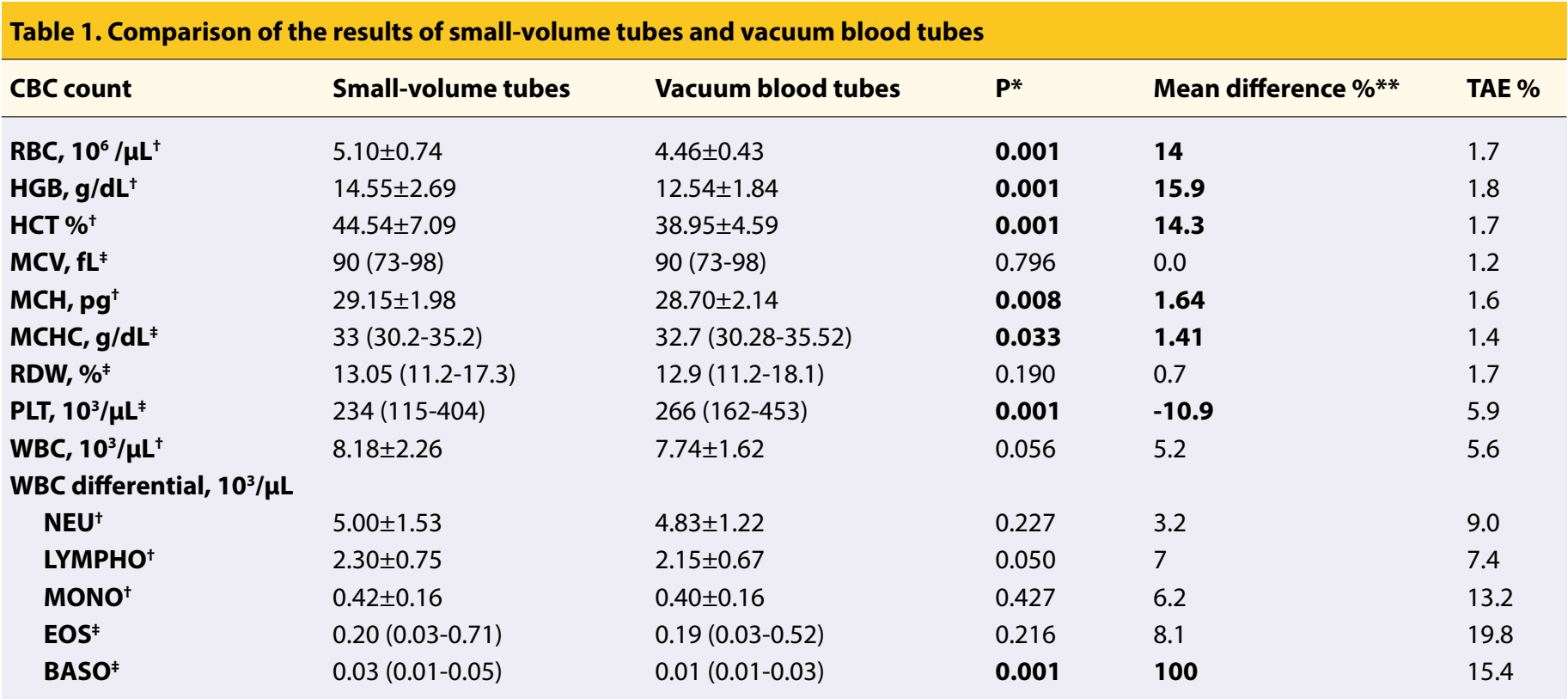

${ }^{\dagger}$ Mean $\pm S D ;{ }^{\ddagger}$ Median (min-max).

* Statistically significant $p$ values are highlighted.

**The values indicating that the mean \% difference exceeded the TAE are highlighted.

BASO: basophil; CBC: complete blood count; EOS: eosinophil; HCT: hematocrit; HGB: hemoglobin; LYMPHO: lymphocyte; MCH: mean corpuscular hemoglobin; MCHC: mean corpuscular hemoglobin concentration; MCV: mean corpuscular volume; MONO: monocyte; NEU: neutrophil; PLT: platelet; RBC: red blood cell; RDW: red cell distribution width; TAE: total allowable error; WBC: white blood cell.

Table 2. Correlation and regression analysis results for small-volume tubes and vacuum blood tubes

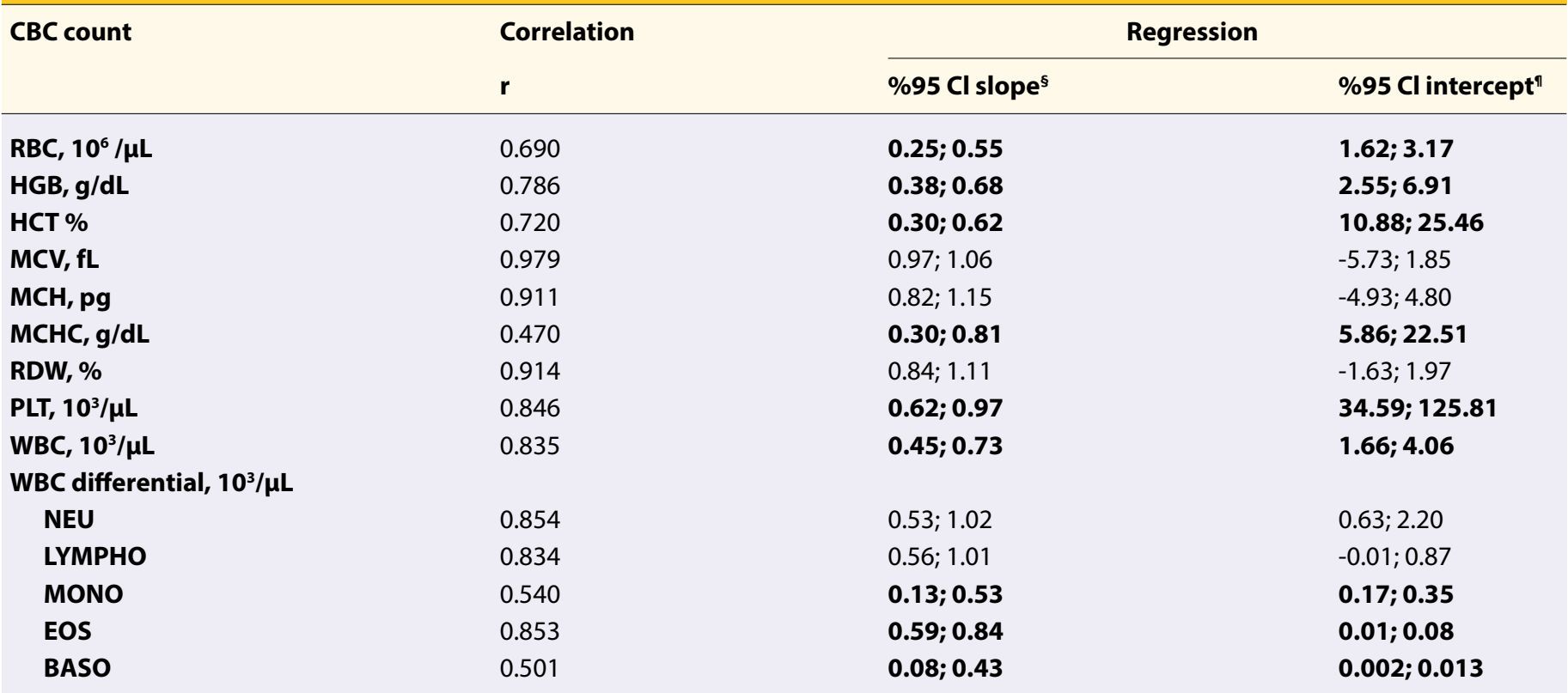

${ }^{5}$ The values for which $95 \% \mathrm{Cl}$ of the slope did not include 1 are highlighted.

"The values for which $95 \% \mathrm{Cl}$ of the intercept did not include 0 are highlighted.

BASO: basophil; CBC: complete blood count; EOS: eosinophil; HCT: hematocrit; HGB: hemoglobin; LYMPHO: lymphocyte; MCH: mean corpuscular hemoglobin; MCHC: mean corpuscular hemoglobin concentration; MCV: mean corpuscular volume; MONO: monocyte; NEU: neutrophil; PLT: platelet; RBC: red blood cell; RDW: red cell distribution width; WBC: white blood cell.

Correlation and regression analysis results of the SVT and vacuum blood tubes are provided in Table 2. A very strong correlation was observed in the MCV, RDW,MCH, NEU, EOS,
PLT, WBC, and LYMPHO levels between the 2 tubes $(r=0.979$, $r=0.914, r=0.911, r=0.854, r=0.853, r=0.846, r=0.835$, and $r=0.834$, respectively). A strong correlation was seen for RBC, 


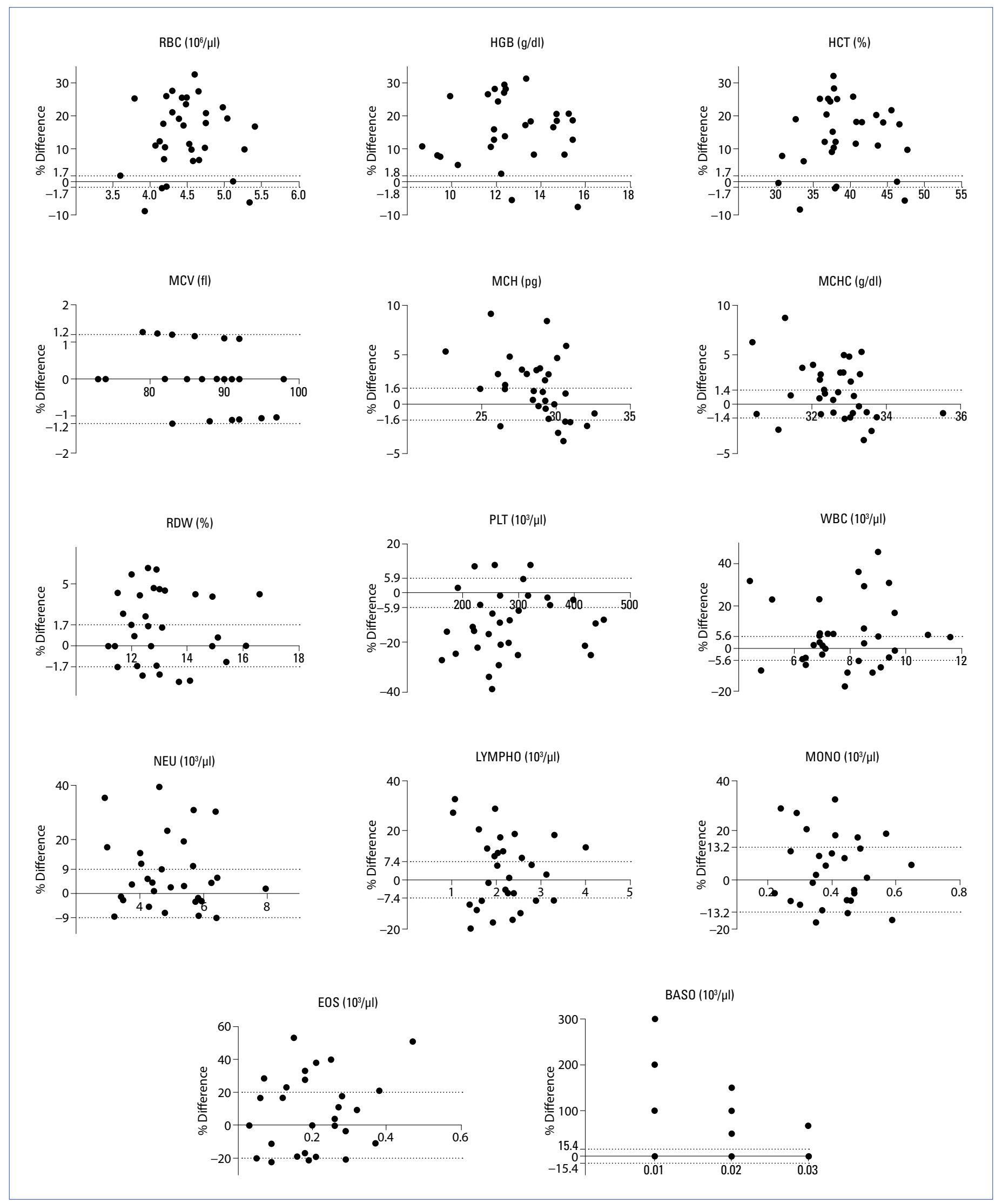

Figure 1. Bland-Altman bias plots comparing the observed difference between BD Microtainer ${ }^{\circledast}$ K2 EDTA tubes vs BD Vacutainer ${ }^{\circledast} 2$ EDTA tubes. The dotted lines delimit the desirable quality specifications (TAE) for bias. 
$\mathrm{HGB}$, and HCT levels ( $r=0.786, r=0.72$, and $r=0.69$, respectively). A moderate correlation was determined for $\mathrm{MONO}, \mathrm{BASO}$, and MCHC levels between the 2 tubes $(r=0.54, r=0.501$, and $r=0.47$, respectively).

Linear regression analysis demonstrated bias for some of the analytes. For RBC, HGB, HCT, MCHC, PLT, WBC, MONO, $E O S$, and BASO, a constant and proportional difference was observed between the 2 tubes evaluated $(95 \% \mathrm{Cl}$ of the intercept does not include 0 and $95 \% \mathrm{Cl}$ of the slope does not include 1). No constant or proportional difference was found in the MCV, MCH, RDW, NEU, and LYMPHO measurements.

Bland-Altman bias plots comparing the observed difference in the results of samples collected in BD Microtainer K2EDTA tubes and the results from samples collected in BD Vacutainer K2EDTA tubes are shown in Figure 1. When the mean percentage difference was compared with the desirable quality specifications (TAE), RBC, HGB, HCT, MCH, MCHC, RDW, WBC, NEU, LYMPHO, MONO, EOS, and BASO values demonstrated a general trend of positive bias, while PLT values demonstrated a general trend of negative bias.

\section{Discussion}

Accurate and effective results are based largely on high quality samples. Taking blood samples from pediatric patients for a CBC can be rather difficult. For newborns, overcollection may cause anemia [13]. Similarly, the impact of blood collection volume requirements has also become more of a concern in geriatric patients [8]. SVTs were developed for CBC assay purposes for these patients [14].

In a study of under-filled blood collection tubes containing K2EDTA used for a CBC, many parameters demonstrated an increased variation when a volume of $0.5 \mathrm{~mL}$ was compared with $4.0 \mathrm{~mL}$ [15]. The authors noted that $1.0 \mathrm{~mL}$ of blood was sufficient for acceptable CBC values. In another study, 4 brands of commercially available capillary blood collection tubes were compared. These tubes were also compared with evacuated blood collection tubes with K2EDTA. In contrast to our study, they found no clinically significant differences between these tubes for $C B C$ use [16]. In the literature, there are several studies that compare capillary blood taken from a SVT and venous blood taken from a vacuum tube; however, the results are conflicting [17-19]. In our study, venous blood collection was used for both tubes.

SVTs can be used to minimize the amount of blood collected. However, there are some disadvantages in the practical use of SVTs: there is a lack of automation for SVTs, they do not have pierceable caps like the larger collection tubes, and the cost of a SVT is much greater than that of a standard tube. Also, $0.5 \mathrm{~mL}$ might be insufficient if a repeat is necessary. These conditions have limited the routine use of SVTs in laboratory medicine. Furthermore, clotted samples have been observed more frequently in SVTs [20].
The design of SVTs may affect the accuracy and usefulness of $C B C$ results. Although other studies have described the effects of SVTs on test results [8, 14,21,22], further research is still needed to explain the effect of SVTs on a CBC. Therefore, the present study compared the performance characteristics of 1 commercially available K2EDTA vacuum blood tube with 1 SVT in a CBC assay. This comparison is highly relevant in order to validate the use of smaller blood volumes for $C B C$. The results of this study indicated that the SVT generated a significant bias in results of $C B C$ assays of venous blood.

Appropriate blood collection is an important pre-analytical step required for reliable test results. Although the influence of blood collection devices on laboratory tests is often ignored, it is essential to perform correct pre-analytical processing. Improper design of blood collection devices can adversely affect the accuracy of laboratory test results [5]. Laboratorians should carefully evaluate the suitability of the means of blood collection and monitor ongoing performance.

\section{Limitations of this study}

The main limitation of this study is the use of healthy adult volunteer blood; therefore, the results of this study cannot be generalized to the overall population. Another important limitation is that due to the insufficient volume in the SVTs, the tubes could not be entered into a stability test.

\section{Conclusion}

This comparison is important to validate the use of smaller blood volumes for $C B C$. The results of this study show that the SVT generated a significant bias in results of CBC.

Conflict of interest: There is no conflict of interest.

Ethics Committee Approval: This study was approved by Ankara Polatlı State Hospital Ethics Committee.

Financial Disclosure: None declared.

Peer-review: Externally peer-reviewed.

\section{References}

1. Buttarello M, Plebani M. Automated blood cell counts: state of the art. Am J Clin Pathol 2008;130:104-16. [CrossRef]

2. Narayanan $\mathrm{S}$. The preanalytic phase. An important component of laboratory medicine. Am J Clin Pathol 2000;113:429-52.

3. Clinical and Laboratory Standars Institute. GP39-A6 Tubes additives for venous and capillary blood specimen collection; Approved Standard. 6th ed. Wayne: Clinical and Laboratory Standards Institute; 2010.

4. Banfi G, Salvagno GL, Lippi G. The role of ethylenediamine tetraacetic acid (EDTA) as in vitro anticoagulant for diagnostic purposes. Clin Chem Lab Med 2007;45:565-76. [CrossRef]

5. Bowen RA, Remaley AT. Interferences from blood collection 
tube components on clinical chemistry assays. Biochem Med (Zagreb) 2014;24:31-44. [CrossRef]

6. Clinical and Laboratory Standars Institute. GP44-A4 Procedures for the Handling and Processing of Blood Specimens for Common Laboratory Tests; Approved Guideline. Wayne: Clinical and Labaratory Standards Institute; 2010.

7. Bowen RA, Hortin GL, Csako G, Otañez OH, Remaley AT. Impact of blood collection devices on clinical chemistry assays. Clin Biochem 2010;43:4-25. [CrossRef]

8. Sanchez-Giron F, Alvarez-Mora F. Reduction of blood loss from laboratory testing in hospitalized adult patients using small-volume (pediatric) tubes. Arch Pathol Lab Med 2008;132:1916-9.

9. Ricós $C$, Alvarez V, Cava F, García-Lario JV, Hernández A, Jiménez $C V$, et al. Current databases on biological variation: pros, cons and progress. Scand J Clin Lab Invest 1999;59:491-500. [CrossRef]

10. Evans JD. Straightforward statistics for the behavioral sciences. Pacific Grove: Brooks/Cole; 1996.

11. Bland JM, Altman DG. Statistical methods for assessing agreement between two methods of clinical measurement. Lancet 1986;1:307-10. [CrossRef]

12. Krouwer JS, Tholen DW, Garber CC, Goldschmidt HM, Kroll MH, et al. Method Comparison and Bias Estimation Using Patient Samples; Approved Guideline-2nd ed. NCCLS document EP9-A2. Wayne: Clinical and Laboratory Standards Institute; 2002.

13. Inal S, Kelleci M. Relief of pain during blood specimen collection in pediatric patients. MCN Am J Matern Child Nurs 2012;37:339-45. [CrossRef]

14. Smoller BR, Kruskall MS, Horowitz GL. Reducing adult phlebotomy blood loss with the use of pediatric-sized blood collection tubes. Am J Clin Pathol 1989;91:701-3. [CrossRef]
15. Xu M, Robbe VA, Jack RM, Rutledge JC. Under-filled blood collection tubes containing K2EDTA as anticoagulant are acceptable for automated complete blood counts, white blood cell differential, and reticulocyte count. Int J Lab Hematol 2010;32:491-7. [CrossRef]

16. Peng Z, Mao J, Li W, Jiang G, Zhou J, Wang S. Comparison of performances of five capillary blood collection tubes. Int J Lab Hematol 2015;37:56-62. [CrossRef]

17. Herrera-Rojas MA, Tarín-Arzaga $L$, Gómez-De León A, Vazquez-Mellado de Larracoechea A, Jaime-Pérez JC, Cantú-Rodríguez OG, et al. No clinically relevant differences between capillary and venous blood cell counts in adult haematological patients using a nonautomated lancet. Int J Lab Hematol 2015;37:e115-8. [CrossRef]

18. Schalk E, Scheinpflug, K, Mohren M. Capillary blood count analyses in clinical practice: a safe, reliable and valid method. LaboratoriumsMedizin 2009;33:303-9. [CrossRef]

19. Schalk E, Scheinpflug K, Mohren M. Correlation of capillary and venous absolute neutrophil counts in adult hematological patients and normal controls. Am J Hematol 2008;83:605.

20. Dillman C, Sandhaus L, Natali R, Baffa A, Forsythe P. A joint pediatric-laboratory quality improvement initiative to reduce pediatric blood sample volumes for laboratory testing: 46. Clinical and Laboratory Haematology 2007;29:37-8.

21. Falch DK. Clinical chemical analyses of serum obtained from capillary versus venous blood, using Microtainers and Vacutainers. Scand J Clin Lab Invest 1981;41:59-62. [CrossRef]

22. Onelöv L, Basmaji R, Svensson A, Nilsson M, Antovic JP. Evaluation of small-volume tubes for venous and capillary PT (INR) samples. Int J Lab Hematol 2015;37:699-704. [CrossRef] 